\title{
The sex differences of the behavior response to early Life immune stimulation: Microglia and astrocytes involvement
}

\author{
I. Berkiks ${ }^{a}$, , L.M. Garcia-Segura ${ }^{b}$, A. Nassiri ${ }^{a}$, A. Mesfioui ${ }^{a}$, A. Ouichou ${ }^{a}$, S. Boulbaroud ${ }^{c}$, \\ Y. Bahbiti ${ }^{\text {a }}$ A.B. Lopez-Rodriguez ${ }^{\mathrm{b}}$, El Hasnaoui ${ }^{\mathrm{a}}$, A. El Hessni ${ }^{\mathrm{a}}$ \\ ${ }^{a}$ Laboratory of Genetic, Neuroendocrinology and Biotechnology, Faculty of Sciences, Ibn Tofail University, Kenitra. Morocco \\ ${ }^{\mathrm{b}}$ Instituto Cajal, C.S.I.C, Madrid, Spain \\ ${ }^{\mathrm{c}}$ Polydisciplinary Faculty, Sultan Moulay Slimane University -Béni Mellal, Morocco
}

\section{G R A P H I C A L A B S T R A C T}

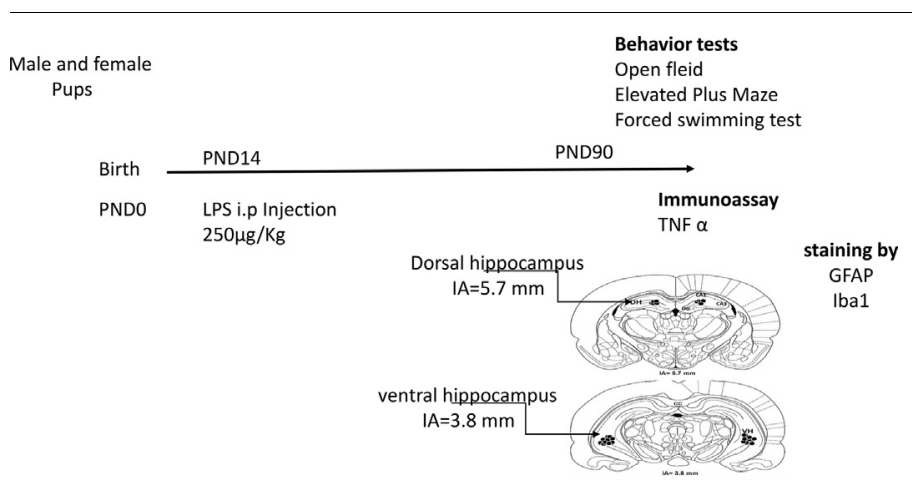

A R T I C L E I N F O

\section{Keywords:}

Anxiety-like

Depression-like

Sex dimorphism

Glial cells

Neuroinflammation

\begin{abstract}
A B S T R A C T
It is well known that inflammatory challenge during the prenatal period results in permanent changes in glial cells and behavior in adulthood. However, it is unknown whether inflammatory challenge during the infantile period may have permanent sexually-dimorphic effects on microglia and astrocytes in vivo, which in turn may be associated with sex differences in adult behavior. In this study, we have evaluated whether postnatal injection of lipopolysaccharide (LPS; $250 \mu \mathrm{g} / \mathrm{kg}$, i.p. on postnatal day 14) induces depressive and less anxiety-like behaviors, glial cell activation, pro-inflammatory cytokine (TNF-alpha) secretion and sexually dimorphic responses in adulthood. Postnatal day 14 (P14) male and female Wistar rats received an intraperitoneal (ip) injection of LPS or PBS. Three months later, animals were tested in the Open Field (OF), the Elevated Plus Maze (EPM) and the Forced Swimming Test (FST) to assess the level of anxiety and depression-like behavior. Hippocampal proinflammatory cytokine TNF-alpha concentration and the number of astrocytes and microglia were estimated in the dentate gyrus, CA1, and CA3 in two regions of the hippocampus (ventral and dorsal). Our results showed that the administration of LPS resulted in less anxiety and depression-like behavior in males but not in females. However, the LPS-administration increased the number of microglia in the dorsal and ventral hippocampus areas in females more than male, while no significant differences in TNF $\alpha$ level had been detected between the LPS-rats treated and their controls. Interestingly, LPS resulted in an increase in the number of astrocytes in both areas of the hippocampus in a female. While in a male, our results showed a decrease in astrocytes number in the dorsal hippocampus, but no significant differences observed in ventral hippocampus.

These findings indicate that an immune challenge in infantile rats induces a ventral and dorsal hippocampus
\end{abstract}

\footnotetext{
* Corresponding author.

E-mail addresses: inssaf.berkiks@uit.ac.ma (I. Berkiks),.1mgs@cajal.csic.es (L.M. Garcia-Segura).
} 
damage in female more than in male, without affecting significantly the affective behavior changes in the female. The results also showed that small changes in the male hippocampus can affect the behavior and induce a depression-like behavior.

\section{Introduction}

Early immune challenges induce long-lasting brain developmental and behavioral impairments and increase the risk of neurodevelopmental and neurodegenerative diseases later in adulthood (Stoll et al. [48]). The activation of the immune system results in the release of proinflammatory cytokines, such as interleukin-1 $\beta$ (IL-1 $\beta$ ), tumor necrosis factor $\alpha$ (TNF- $\alpha)$ ([3]; Berkiks. I et al. [4]) and IL-6 [1]. High levels of these cytokines during development are associated with low resilience to diseases in adulthood $[8,44,58]$.

Sex steroids, such as estradiol, testosterone, and progesterone play important roles in immune system regulation [10,32,45]. In general, estradiol increases [17] while testosterone decreases peripheral immune response [46] and females show more pronounced responses than males to immune activation. Sex steroids also influence hypothalamuspituitary-adrenal (HPA) axis activity, since corticotrophin-releasing hormone (CRH) production is positively modulated by estrogens [51] and negatively modulated by androgens [6]. Consequently, females exhibit higher plasma corticosterone levels compared to males. Furthermore, lipopolysaccharide (LPS) administration in mice induces an increase of plasma corticosterone levels in females than in males [46]. During the postnatal developmental period, glial cells play an essential role in the organization of synaptic circuits by engulfing synaptic material and eliminating synapses [36,59]. Microglia and astrocytes also participate in the generation of sexually-dimorphic synaptic circuits during this period $[15,28]$. Therefore, the alteration of microglia during this period may have long-lasting consequences for brain function and for brain sex differentiation, especially in the hippocampus.

The hippocampus is the area that is mainly involved in mood and emotion changes. It is divided into three compartments: dorsal, intermediate and ventral hippocampus [13]. Several studies demonstrated that the lesion in hippocampus able to induce an impairment several types of memory suffer, including episodic memory and spatial learning. The dorsal hippocampus is primarily responsible for cognitive functions [11]. The ventral hippocampus is related more to stress, anxiety, and depression-like behavior [13].

However, it is unknown whether an inflammatory challenge during the infantile period may have permanent sexually-dimorphic effects in microglia and astrocytes in the dorsal and ventral hippocampus, which in turn may be associated with sex differences in adult behavior. Therefore, the present investigation was designed to assess whether administration of LPS to infantile rats results in sex-dimorphic permanent changes in microglia and astrocytes within the ventral and dorsal hippocampus in adult life and whether these changes are correlated with sex-dimorphic alterations in affective behavior.

\section{Material and methods}

\subsection{Animals and experimental treatments}

Pregnant Wistar rats (Genetic - Neuroendocrinology Biotechnology Laboratory located at Ibn Tofail University (Kenitra, Morocco) were maintained at a constant temperature $\left(24^{\circ} \mathrm{C}\right)$ with a relative humidity of $50-60 \%$. A 12-h dark/light cycle was maintained with lights on between 7:00 p.m. and 7:00 a.m. Food and water were available ad libitum. Pups were randomly distributed among mothers so that each mother had the same number of pups (eight pups per dam and the same ratio of male to female pups). 14 days old pups ( $n=8$ / group) were given a single i.p injection of LPS $(250 \mu \mathrm{g} / \mathrm{kg}$, Escherichia coli, serotype 026: B6, L-3755, Sigma, St. Louis MO) to induce a chronic inflammation until adulthood or an equivalent volume of PBS. The pups were weaned at P21 and males and females were separated. Animals were housed in groups of four per plexiglass cage $(430 * 290 * 210 \mathrm{~mm})$ until 90-97 days of age when the behavioral tests were carried out.

\subsection{Ethics committee}

All experimental procedures were performed according to the $\mathrm{NIH}$ Guide for the Care and Use of Laboratory Animals and following the doctoral education center Guide at IBN TOFAIL university.

\subsection{Behavioral analysis}

\subsubsection{Estrous cycle tracking}

At approximately P90, the afternoon assessment of estrous cycles began. Vaginal smears were collected daily. Female rats were cycling normally (4-5 d cycles). Two weeks before, all the females were placed in the same place, 4 females per cage for synchronizing the cycles and being assigned to a group to represent one of three phases of the estrous cycle (diestrus, proestrus, or estrus). Once synchronized and assigned to an estrous phase group; the animals were passed to the behavioral tests.

\subsubsection{Open-field}

The investigatory behavior was tested in a wooden apparatus $(100 \mathrm{~cm} \times 100 \mathrm{~cm})$. As previously reported [12], it was enclosed by $40 \mathrm{~cm}$ high walls and placed under strong illumination (100 watts, $2 \mathrm{~m}$ above the apparatus). The area was divided into 25 squares $(20 \mathrm{~cm} \times 20 \mathrm{~cm})$, defined as nine central and sixteen peripheral squares. At the beginning of the 10-min test, the animal was placed in the center of the apparatus and its behavior was videotaped for subsequent analysis. The device was cleaned after each individual rat session. The quantified parameters used in this experiment was the total number of squares visited [38].

\subsubsection{Elevated plus-maze}

The EPM is an ethological model of anxiety-like behavior in rodents provoked by the novelty and repulsion as a result of elevation and illumination of the maze [52]. This test is based on the creation of a conflict between the exploratory drive of the rat and its innate fear of open and exposed areas; it has been validated for the detection of emotional responses to anxiogenic and anxiolytic substances. Thus, increased open-arm exploration indicates reduced anxiety-like-related behavior. The EPM consists of a wooden plus-shaped platform elevated $70 \mathrm{~cm}$ above the floor. Two of the opposing arms $(50 \mathrm{~cm} \times 10 \mathrm{~cm})$ are closed by $40 \mathrm{~cm}$-high sides and end walls, having an open roof. In order to avoid fall, the other two arms (open arms) were surrounded by a $0.5 \mathrm{~cm}$ high edge. The four arms had, at their intersection, a central platform $(10 \mathrm{~cm} \times 10 \mathrm{~cm})$. A 100 -W lamp was placed exactly on the central platform. At the beginning of the test, the rats were placed in the central area of the maze facing an open arm. Behavior was videotaped for $5 \mathrm{~min}$ for time spent in each section of the maze and number of entries. Time spent in the arms and the number of entries was used to compute time and entries ratio (open/closed) that were used for analysis. Decreased anxiety-like behavior is illustrated by a significant statistical increase of parameters in open arms (time and/or entries). Although total entries reflect the motor component of the exploratory activity.

\subsubsection{Forced swimming test}

Swimming sessions were conducted by placing the rat in individual 
glass cylinders $(50 \mathrm{~cm}$ in height and $30 \mathrm{~cm}$ in diameter) containing $30 \mathrm{~cm}$ of water at $\left(23 \pm 2{ }^{\circ} \mathrm{C}\right)$. During the session, rats were forced to swim for $5 \mathrm{~min}$ and the duration of immobility was measured. The latency to the first period of immobility was also recorded starting immediately after placing the rats in the cylinder. A rat was judged immobile when it ceased all active behaviors (i.e. struggling, swimming, and jumping) and remained passively floating or making minimal movements necessary to maintain the nostrils above water. A high percentage of time floating is interpreted as an increased depressivelike response [18]. The animals were placed into the testing apparatus under illumination (100 watts). All behaviors were recorded in a video for subsequent analysis [9].

\subsection{TNF $\alpha$ assay}

After the completion of the behavioral analysis, animals were deeply anesthetized with chloral hydrate $(100 \mathrm{mg} / \mathrm{kg})$. Brains were removed and the hippocampal region of each hemisphere was dissected out and homogenized in ice-cold lysis buffer (RIPA lysis buffer $+1 \mathrm{mM}$ PMSF) using a Dounce homogenizer. The homogenates were centrifuged $\left(15 \mathrm{~min}\right.$ at $14000 \mathrm{~g}$ ) and stored at $-80^{\circ} \mathrm{C}$. Protein content was assayed by Bradford procedure. The TNF $\alpha$ content of the samples was assayed using a rat TNF $\alpha$ ELISA kit (Invitrogen KRC3011). The TNF $\alpha$ content was expressed as picogram of cytokine per milliliter of homogenate.

\subsection{Immunohistochemistry}

Immunohistochemistry was carried out on free-floating sections for dorsal and ventral hippocampus under moderate shaking. All washes and incubations were done in $0.1 \mathrm{M}$ phosphate buffer $\mathrm{pH} 7.4$, containing $0.3 \%$ bovine serum albumin and $0.3 \%$ Triton X-100. The endogenous peroxidase activity was quenched for $10 \mathrm{~min}$ at room temperature in a solution of $3 \%$ hydrogen peroxide in $30 \%$ methanol. After several washes in buffer, sections were incubated overnight at $4{ }^{\circ} \mathrm{C}$ with a monoclonal antibody for ionized calcium-binding adapter molecule 1 (Iba1) (diluted 1:2000), a marker of microglia, or a monoclonal antibody for glial fibril acid protein (GFAP) (diluted 1:1000; Clone GA5, Sigma-Aldrich), a marker of astrocytes. Sections were then rinsed in buffer and incubated for $2 \mathrm{~h}$ at room temperature with biotinylated goat anti-mouse immunoglobulin G (diluted 1:300; Pierce). After several washes in buffer, sections were incubated for $90 \mathrm{~min}$ at room temperature with avidin-biotin-peroxidase complex (diluted 1:250; ImmunoPure $\mathrm{ABC}$ peroxidase staining kit). The reaction product was revealed by incubating the sections with $2 \mathrm{mg} / \mathrm{mL}$ 3,3-diaminobenzidine (Sigma-Aldrich) and $0.01 \%$ hydrogen peroxide in $0.1 \mathrm{M}$ phosphate buffer. Then, sections were dehydrated, mounted on gelatinized slides, coverslipped, and examined with a Leica DMRB-E microscope.

\subsubsection{Glial cell counts}

All morphometric analyses were performed on coded sections. The number of immunoreactive cells was estimated by the optical dissector method [24] using total section thickness for dissector height [20] and a counting frame of $55355 \mathrm{~lm}$. Total of 78 counting frames was assessed per animal and per each zone analyzed. Section thickness was measured using a digital length gauge device (Heidenhain-Metro MT 12/ND221) attached to the stage of a Leica microscope. Cell nuclei from immunoreactive cells that came into focus while focusing down through the dissector height were counted. As an alternative method to evaluate glial reactivity, the surface density of Iba1 and GFAP immunoreactive cell bodies and cell processes were assessed in 3 DG, CA1, and CA3 of the hippocampus. The ratio of the surface of immunoreactive profiles to the reference volume (surface density, Sv) was calculated by the following formula: $\mathrm{Sv}=2 \mathrm{I} / \mathrm{L}$, where $\mathrm{I}$ is the number of points at which the immunoreactive profiles (GFAP immunoreactive astroglial cell bodies and processes) cross the test grid lines and $\mathrm{L}$ is the test line length in the tissue [54]. The test grid used is based on the C16 grid of Weibel [54] and has 10310 lines of a total length of $2000 \mathrm{~lm}$ $(\mathrm{L}=2 \mathrm{~mm})$. Magnification was calculated with a calibrated slide $(100$ lines/mm, Leitz). For each animal, a minimum of 2 sections was evaluated.

All immunoreactive profiles, whether heavily or less heavily labeled, were considered for quantification.

\subsection{Data analysis}

All the data were analyzed using a two-way analysis of variance (ANOVA) followed by Bonferroni 's post hoc test for comparison between groups. All tests were used with significance set at ${ }^{*} p<.05$, $* * p<.01, * * * p<.001$ and the data were presented as a mean \pm standard error of the mean.

\section{Results}

\subsection{Behavioral tests}

\subsubsection{Open field}

We used the OF test to assess locomotor activity three months after the postnatal exposure to LPS $(250 \mu \mathrm{g} / \mathrm{Kg})$. LPS induced i) no significant changes in the number of total squares visited by female and male rats after 95 days from LPS injection $(F(1,28)=1,52, P=0,2281)$, ii) no significant interaction between sex and LPS $(F(1,28)=4,00$, $\mathrm{P}=0,0553)$ and iii) no sex significant differences was revealed ( $\mathrm{F}(1$, $28)=0,594, P=0,4475)$, Fig. 1 . We can conclude that postnatal immune stimulation has no effect on the locomotor activity of male and female rats in open field test.

\subsubsection{Elevated plus maze}

The results in Fig. 2.A.B show that neonatal LPS exposure did not affect the locomotor activity in the Elevated plus maze test. The statistical analysis of the total entries into the arms showed no significant effect of treatment $(\mathrm{F}(1,28)=1.62, p=.22)$, no sex differences ( $\mathrm{F}$ $(1,28)=0.66, p=.42)$ and no interaction between treatment and sex $(\mathrm{F}(1,28)=0.000, p=1)$ Fig. 2A. Fig. 2B shows that the percent of entries in the open arms was also unaffected, with no effect of treatment $(\mathrm{F}(1,28)=0.09, p=.77)$ or $\operatorname{sex}(\mathrm{F}(1,28)=0.05, p=.821)$ and no interaction between factors $(\mathrm{F}(1,28)=1.10, p=.304)$. Male rats injected with LPS showed a significant increase in the time spent in open arms compared with control male rats while the females injected with LPS had a decrease in time spent in open arms (Fig. 2.C). The post hoc test

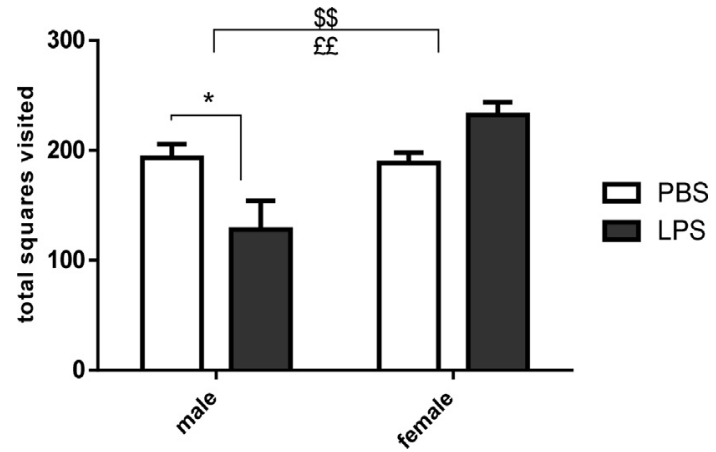

Fig. 1. Effects of i.p. injection of LPS $(250 \mu \mathrm{g} / \mathrm{kg})$ on locomotor performances at 90/97 days post-treatment in open field test. $n=8$ /group. Two way ANOVA with treatment and gender as factors revealed a significant difference in the interaction between the sex and LPS $(F(1,28)=11, P=0,0025)$, a sex significant differences $(\mathrm{F}(1,28)=9.25, \mathrm{P}=0,0051)$ and no significant effect of LPS on locomotor activity $(\mathrm{F}(1,28)=0.448, \mathrm{P}=0,51)$. All data expressed as mean \pm SEM, ${ }^{*} p<.05$ treatment impact, $£ \mathrm{p}<.05$ interaction between the sex and treatment, $\$ \mathrm{p}<.05$ the sex impact. 

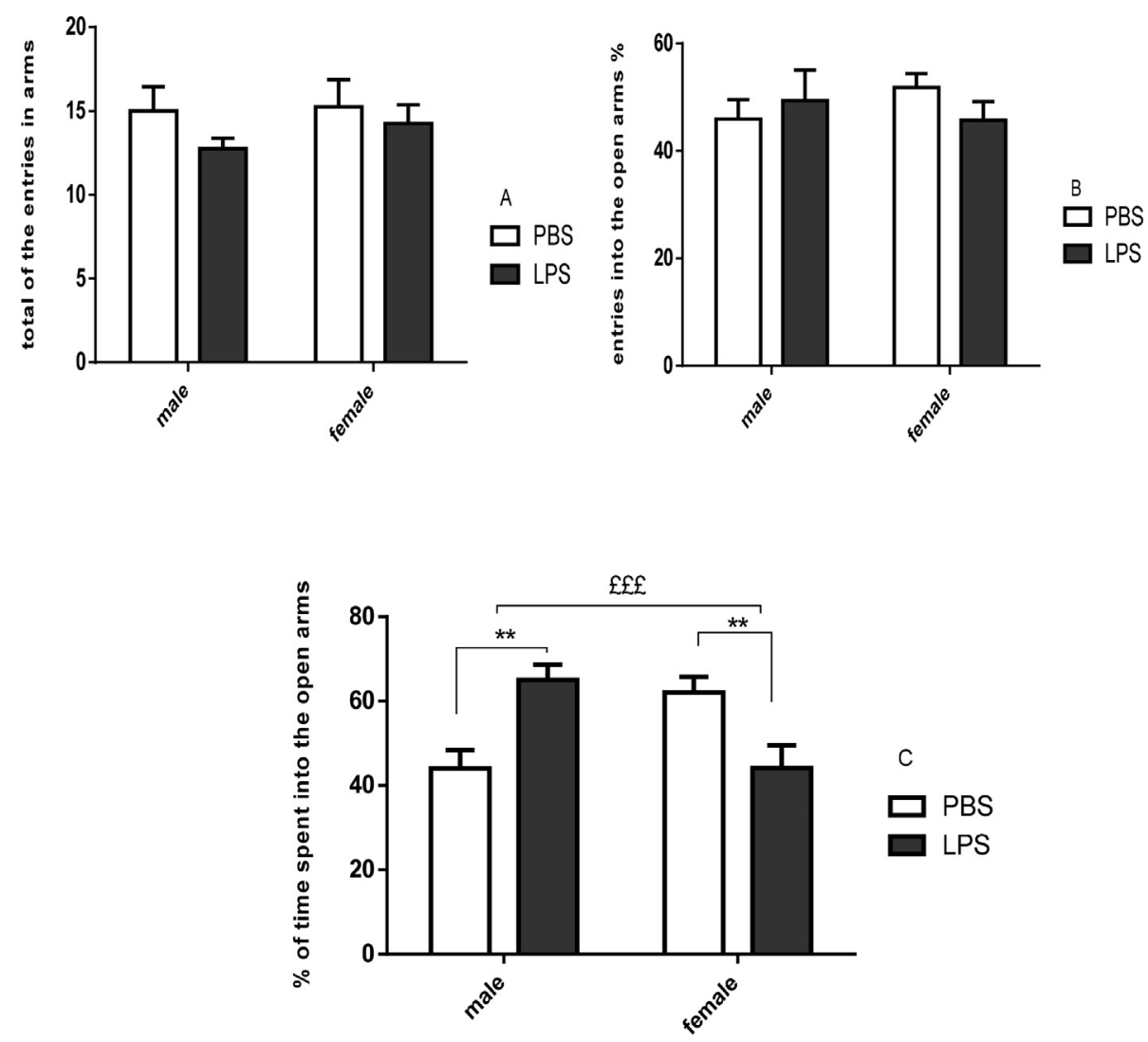

revealed a significant effect of treatment $(\mathrm{F}(1,28)=0.44, p=.041)$ no sex impact are observed in the performance $(\mathrm{F}(1,28)=0.01, p=.92)$ and very significant interaction between sex and LPS injection ( $F$ $(1,28)=11.55, p=.0037)$. (Fig. 2.C).

\subsubsection{Forced swimming test}

FST was used to evaluate depressive-like behavior. Fig. 3 shows that LPS injection did not affect the time of immobility in female rats compared to control rats. In contrast, LPS treatment significantly increased the immobility time in male rats (Fig. 3) compared to control rats. Furthermore, Fig. 3 shows that female controls showed a higher time of immobility in FST than male controls (Fig. 3).

The results of the forced swimming test show that neonatal LPS exposure increases the duration of immobility time in male but not female rats compared with their respective controls. These results revealed more depressive-like behavior in male rats that were postnatally exposed to LPS taking into consideration that LPS had no effect on locomotor activity measured by OF. Two-way ANOVA followed by Bonferroni test revealed a significant effect of treatment (F $(1,28)=23.7, p=.0002)$ but no sex effect $((\mathrm{F}(1,28)=2.67, p=.124)$, and a high significant interaction between LPS and sex response ( $\mathrm{F}$ $(1,28)=10.02, p=.0069)$.

\subsubsection{TNFa levels in the hippocampus}

The levels of neural pro-inflammatory cytokine TNF $\alpha$ were assessed in the whole hippocampus 3 months after injection of LPS or PBS to determine the persistence of inflammation until adulthood. The postnatal administration of LPS showed no significant increase in the levels of hippocampal TNFa, both in female and male rats compared to their controls at the adult age (Fig. 4). The same analysis showed that females exhibited an increase in hippocampal levels of TNF $\alpha$ than males (Fig. 4). The two-way ANOVA revealed that sex significantly affects the TNF $\alpha$ production in hippocampus $(\mathrm{F}(1,16)=33,789 ; p=.0001)$ but not treatment $(\mathrm{F}(1,16)=0.07, p=.136)$ and no interaction significant
Fig. 2. Effects of i.p. injection of LPS on anxiety-like behavioral performances at 90 days post-treatment in elevated plus maze in male and female.(A) total entries (in this parameters the rats males and females performances are not affected by early life LPStreatment $\mathrm{F}(1,28)=1,62 ; \mathrm{P}=0,2211)$, (B) entries into open arms, ANOVA revealed no significant differences in the male and female rats-treated by LPS performance compared to their controls ( $F$ (1, 28 ) $=0,0879 ; \mathrm{P}=0,7707$ ) (C) time into open arms, showed a significant differences in the interaction between treatment and gender $(F((1,28)=11,6$, $\mathrm{P}=0,0037$ ), while the male LPS-treated had a significant increase in the time spent in the open arms $p=.04 . \mathrm{n}=8$ /group. * $\mathrm{p}<.05$ treatment compared with corresponding control group, $£ £ p<.01$ interaction between sex and treatments compared with corresponding control groups (two way ANOVA and followed by Bonferroni test during the group's comparisons) all data expressed as mean \pm SEM.
$(\mathrm{F}(1,16)=3.47, \mathrm{p}=.136)$

\subsection{Glia analysis}

\subsubsection{Astrocyte analysis}

The number of GFAP-immunoreactive astrocytes in the dorsal dentate gyrus results showed no treatment effect $(F(1,16)=5.33$, $P=0,08)$ no sex differences were detected $(\mathrm{F}(1,16)=1.33, \mathrm{P}=0$, 31 ) nor any interaction between LPS and $\operatorname{sex}(F(1,16)=0.000, P=1)$. The results in dorsal CA1 showed a significant difference in treatment effect $(F(1,16)=203, P=0,0001)$ sex effect $(F(1,16)=61,1$. $\mathrm{P}=0,0014)$ and an interaction between treatment and sex ( $\mathrm{F}(1$, 16) $=73,2$. $P=0,0010) \quad($ Fig. 5). The dorsal CA3 GFAP-

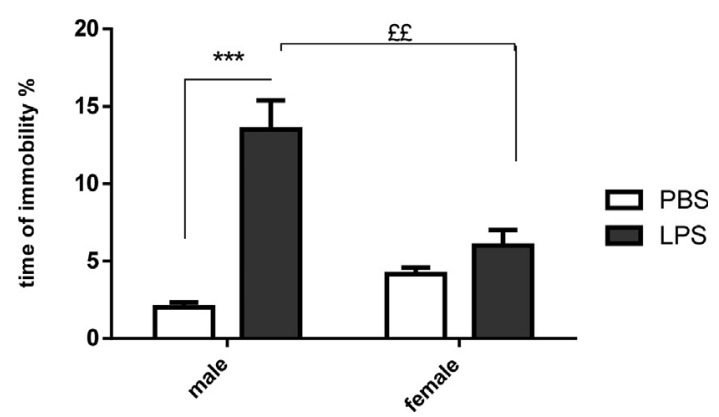

Fig. 3. Effects of i.p. injection of LPS on depression-like behavioral performances at 90 days post-treatment, the immobility time. The results showed a significant difference in the interaction between the LPS-treatment and gender, $(F(1,28)=10,0 ; P=0,0069)$. Furthermore a significant differences in LPStreated rats compared to the control $(\mathrm{F}(1,28)=23,7 ; \mathrm{P}=0,0002) . \mathrm{n}=8$ / group. ${ }^{* * *} p<.001$ treatment compared with corresponding control group (two way ANOVA followed by Bonferroni test), and $£ £ \mathrm{p}<.01$ treatment compared with corresponding control group (two-way ANOVA test) all data expressed as mean \pm SEM. 


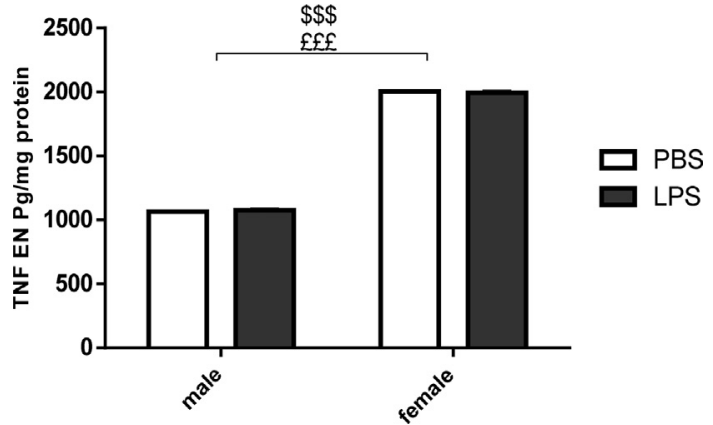

Fig. 4. Effects of i.p. injection of LPS on hippocampus TNF $\alpha$ expression at 90 days post-treatment. $n=5$ /group. $\$ \$ \$$ indicate the gender effect in the basal level of TNF $\alpha(\mathrm{F}(1,16)=101,640 ; P<0,0001)$, and $£ £ £$ indicate the interaction between LPS and gender on the level of Hippocampal TNF $\alpha$ after 90 days from the injection with $(\mathrm{F}(1,16)=10,4 ; \mathrm{P}=0,0073)$. (Two way ANOVA test) all data expressed as mean \pm SEM. Interaction sex.

immunoreactive results showed significant differences in treatment effect $(\mathrm{F}(1,16)=80,90 . \mathrm{P}=0,0008)$ no significant in sex effect $(\mathrm{F}(1$, 16) $=6494, P=0,0634$ ) with an interaction between sex and treatment $(F(1,16)=14,04 . P=0,0200)$.

In a ventral hippocampus, the results showed a significant increase in the number of astrocytes after 85 days from the LPS injection in a female (Fig. 6), while in male LPS injected the results showed no significant differences compared to the control male. The statistics results showed that in DG, the treatment by LPS significantly affected the number astrocytes $((\mathrm{F} .16)=16.03, p=.0161)$ significant differences between sex $((\mathrm{F} .16)=37.12, p=.0037)$ and no interaction between the LPS and sexes $(\mathrm{F} .16)=5.2, p=.084)$. The CA1 results showed a significant effect of treatment, the sex differences in the number of astrocytes and the interaction between the LPS and sexes $((\mathrm{F} .16)=85.33, \quad p=.0008), \quad(\mathrm{F} .16)=85, \quad p=.0007)$, $(\mathrm{F} .16)=25.04, p=.00075)$. The statistics astrocytes results in ventral CA3 showed a significant effect of treatment (F.16) $=75, p=.0001)$ no sex differences in astrocytes number (F.16) $=0.33, p=.59$ ) and a significant interaction between LPS and sexes (F.16) $=34.38, p=.0042$ ).

As a summary, our results showed that rats-LPS treated showed a significant increase in the number of astrocytes in CA1 and CA3, but not in the dentate gyrus, compared to control animals. This effect of LPS was observed in both of sexes. However, in CA1 and CA3, the number of astrocytes was significantly higher in LPS treated females than in the LPS treated males (Fig. 6).

\subsubsection{Microglia analysis}

The dorsal hippocampus microglia results showed in DG a significant effect of LPS on the microglia cells number in this area (F $(1.16)=74, p<.0001)$, with a significant sex effect $(\mathrm{F}(1.16)=16.1$, $p<.0039$ ) and a high interaction between LPS and sex (F $(1.16)=8.22, p<.02$ ) (Fig. 8). In the same area, the results of reactive microglia show that LPS could activate significantly the cells (F $(1.16)=22.9, p<.0014)$ with no sex differences observed (F $(1.16)=0.08, p<.78)$ and no interaction between sex and LPS (F $(1.16)=0.52, p<.48)$. In CA1 hippocampal area, we observe no significant effect of LPS injection on microglia number $(\mathrm{F}(1.16)=5.94$, $p=.050)$, no sex effect $(\mathrm{F}(1.16)=3.61, p=.10)$ and no interaction sex treatment $(\mathrm{F}(1.16)=2.18, p=.19)$.

While the activation microglia results in the same area showed a significant impact on LPS $(\mathrm{F}(1.16)=90, p=.0007)$ without any sex differences observed $(\mathrm{F}(1.16)=0.0474, p<.84)$ and any interaction existed $(\mathrm{F}(1.16)=1.77, p<.25)$ (Fig. 7$)$, the results of reactive microglia observed in CA3 showed a significant impact on LPS (F $(1.16)=37.7, p<.0036)$ without any sex differences observed $(\mathrm{F}$
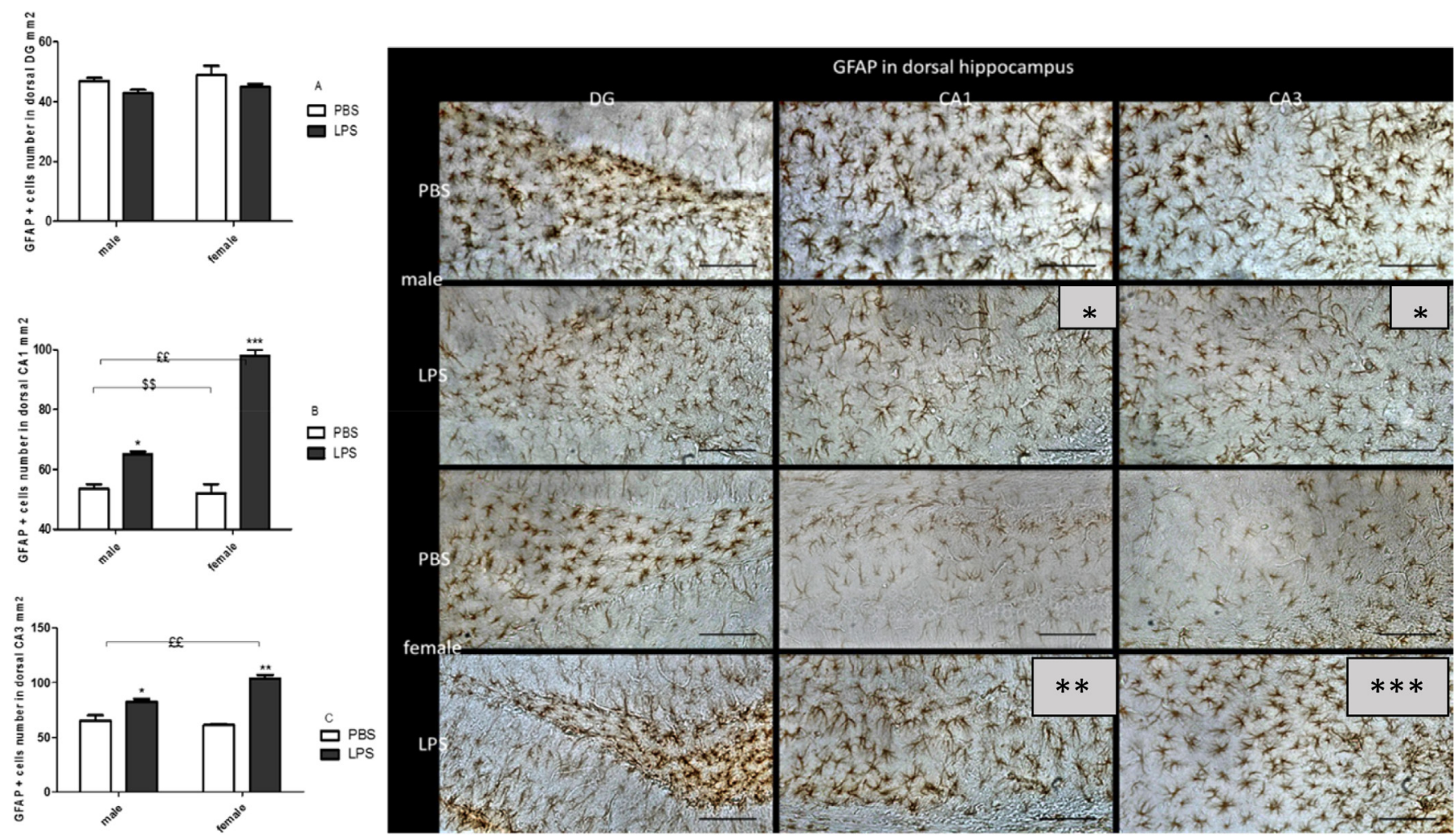

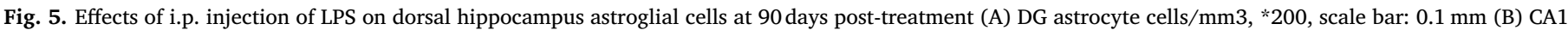

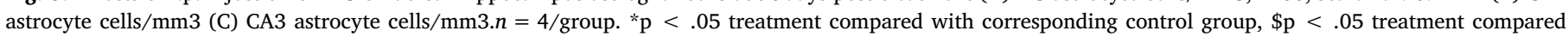

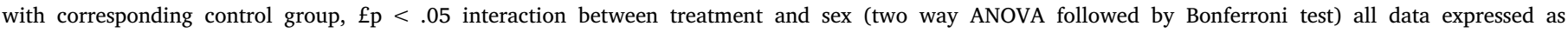
mean \pm SEM. 

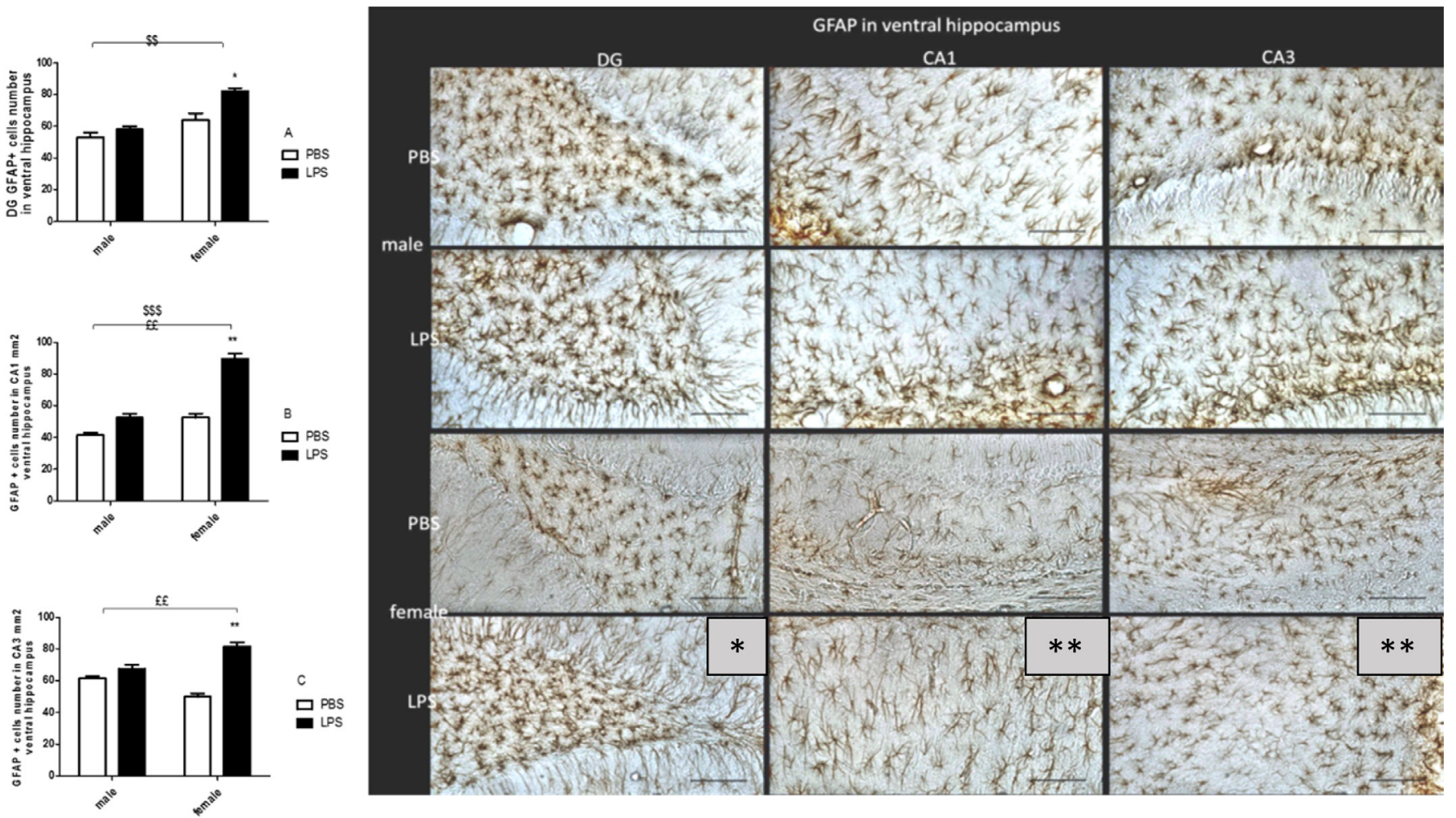

Fig. 6. Effects of i.p. injection of LPS on the ventral hippocampus astrocyte cells numbers at 90 days post-treatment, *200, scale bar: $0.1 \mathrm{~mm}$ (A) DG astrocyte cells/ $\mathrm{mm} 3$, (B) CA1 astrocyte cells $/ \mathrm{mm} 3$ (C) CA3 astrocyte cells/mm2.n $=4$ /group. *p $<.05$, **p $<.01, * * * \mathrm{p}<.001$ treatment compared with corresponding control group, $\$ \mathrm{p}<.05, \$ \$ \mathrm{p}<.001$ treatment compared with corresponding control group, $\mathrm{fp}<.05, £ £ \mathrm{p}<.01$ interaction between treatment and sex (two way ANOVA followed by Bonferroni test) all data expressed as mean \pm SEM.

$(1.16)=0.065, p<.81)$, and any interaction existed between LPS and $\operatorname{sex}(\mathrm{F}(1.16)=0.89, p<.39)$ Fig. $5 \mathrm{C}$

The results of the microglia in the ventral hippocampus (Fig. 8) showed that in DG a significant effect of LPS on the microglia activation in this area $(\mathrm{F}(1.16)=76.4, p<.0009)$ with no significant sex effect $(\mathrm{F}(1.16)=0.005, p<.94)$ and a high interaction between LPS and sex $(\mathrm{F}(1.16)=10.7, p<.03) \mathrm{Fig}$. 6.A. In CA1 we observe a highly significant effect of LPS injection on microglia activation $(\mathrm{F}(1.16)=168$,
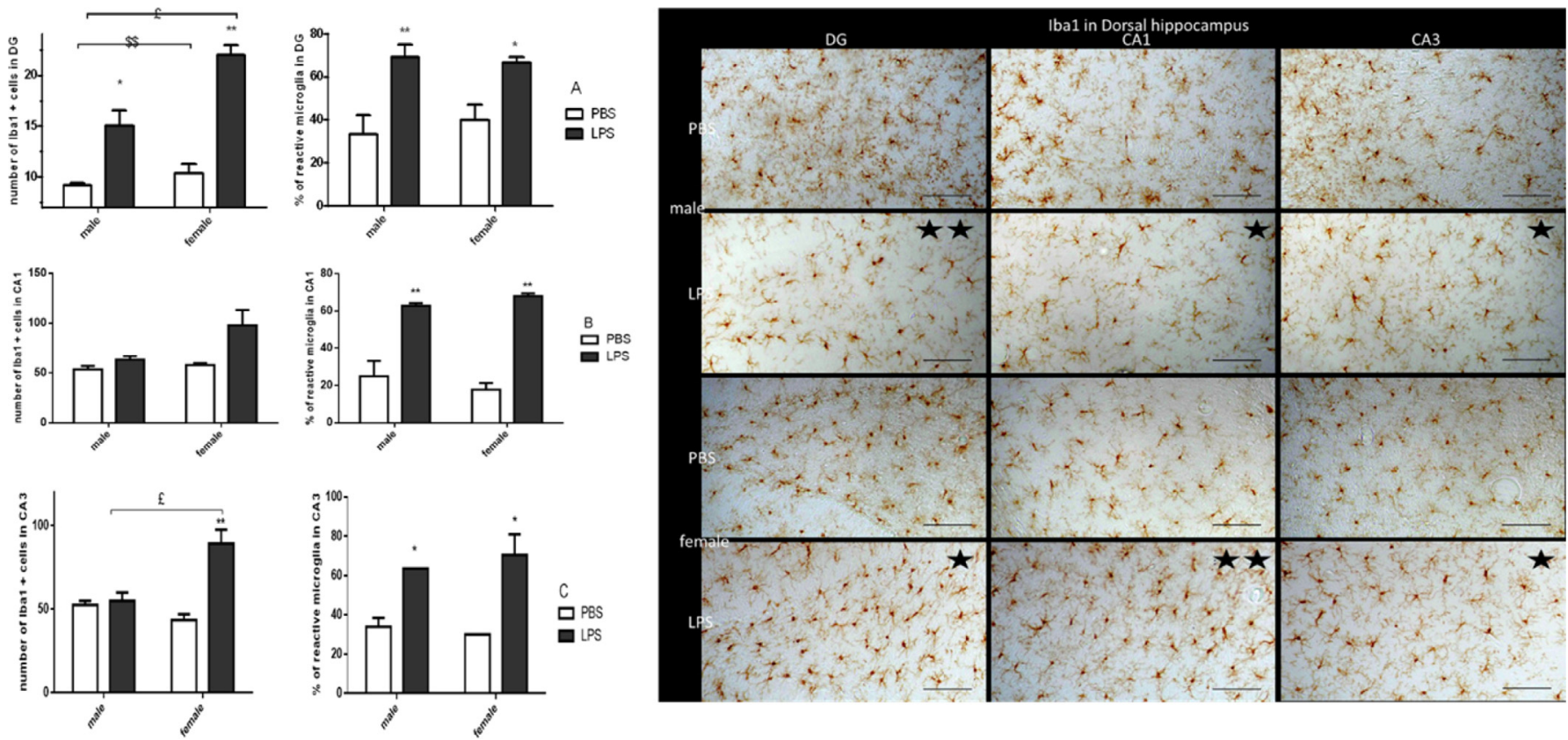

Fig. 7. Effects of i.p. injection of LPS on the number/activation dorsal hippocampus microglial cells at 90 days post-treatment (A) DG microglia cells/mm3, *200, scale bar: $0.1 \mathrm{~mm}$ (B) CA1 microglia cells $/ \mathrm{mm} 3$ (C) CA3 microglia cells $/ \mathrm{mm} 3 . \mathrm{n}=4 /$ group. ${ }^{*} \mathrm{p}<.05$ treatment compared with corresponding control group, $\$ \mathrm{p}<.05$ treatment compared with corresponding control group, $\mathrm{fp}_{\mathrm{p}}<.05$ interaction between treatment and sex (two way ANOVA followed by Bonferroni test) all data expressed as mean \pm SEM. 

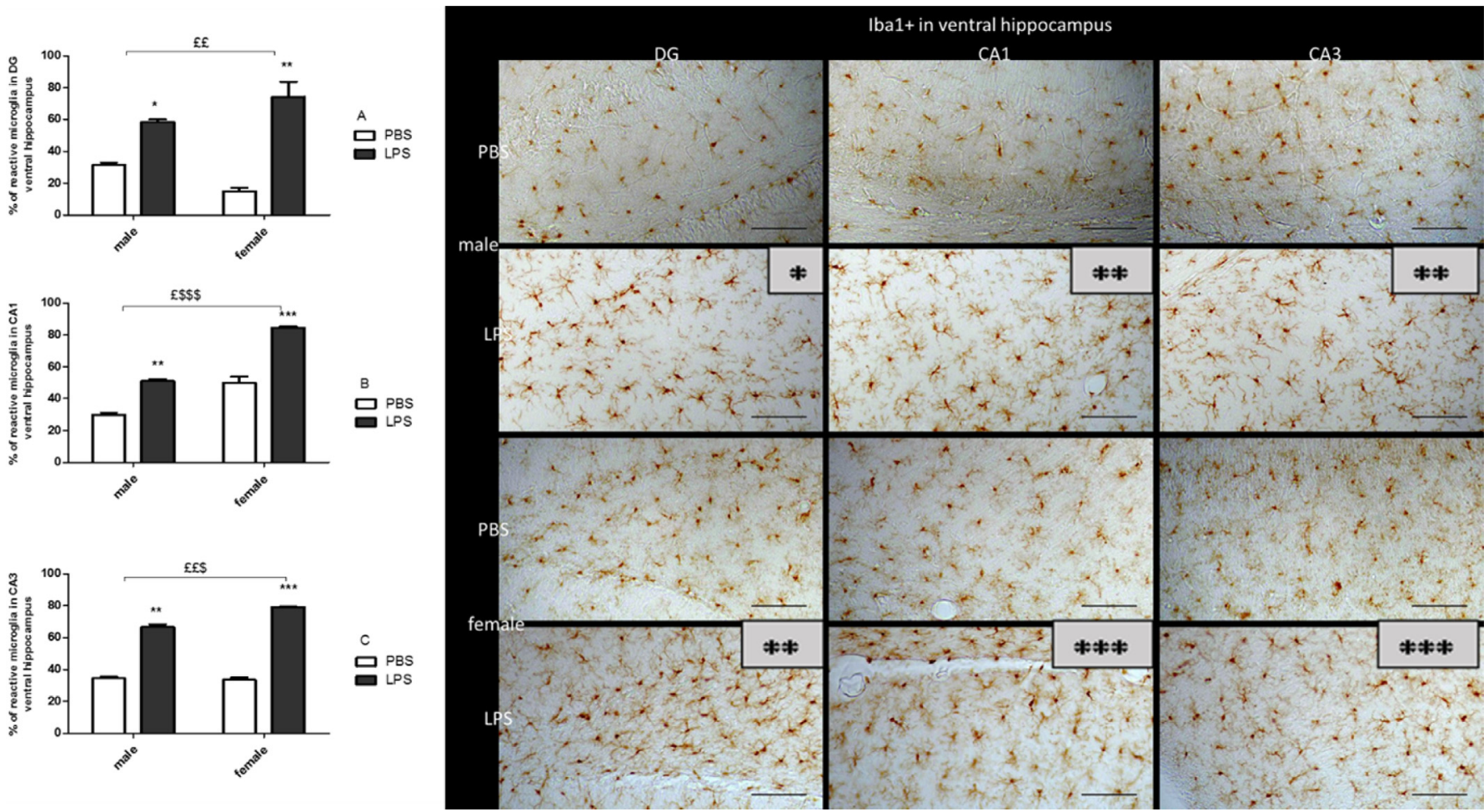

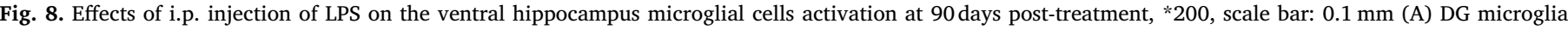

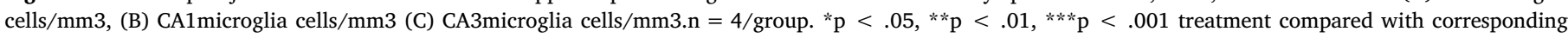

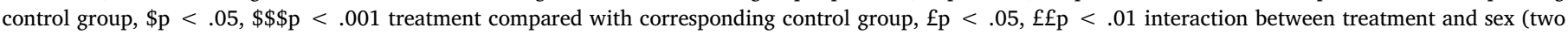
way ANOVA followed by Bonferroni test) all data expressed as mean \pm SEM.

$p=.0002)$ with high sex effect $(\mathrm{F}(1.16)=155, \mathrm{p}=.0002)$ and interaction between sex and treatment $(\mathrm{F}(1.16)=9.65, p=.03)$. The same microglia activation results we obtained in CA3 ventral area with observing a highly significant effect of LPS injection on microglia activation $(\mathrm{F}(1.16)=886, p=.0000)$ with sex effect $(\mathrm{F}(1.16)=19.2$, $p=.010)$ and interaction sex treatment $(\mathrm{F}(1.16)=26.7, p=.0067)$ (Fig. 8).

\section{Discussion}

The present study indicates that the immune challenge in infantile rats (PND14) caused sexually-dimorphic behavioral alterations in adulthood (PND 90). Infantile male rats injected with LPS (male-LPS treated) presented a less locomotor activity in OF, less anxiety-like in EPM and more depression-like behaviors in FST than females-LPS treated, compared to their controls. These results suggest that depression- and anxiety-related phenotypes can be dissociated in rats-LPS models and that the early life immune stimulation might affect brain circuits and behaviors differently although they overlap each other. Those two phenotypes are more likely related to the sickness behavior resulting from infantile immune stimulation, which probably persists until adult age [31].

One of the possible reasons for the significant increases in the time spent into the open arms may be due to the learning deficiency in male LPS-treated rats. In the same context, Wang and his colleagues demonstrated that neonatal LPS exposure caused no significant differences in male and female rats treated in the neonatal period with LPS [53]. The hippocampal injury may induce learning and memory deficits in both male and female rats. Wang attributed the behavioral change to the sex differences in the colonization and function of glia between males and females [53]. The most likely explanation is that the inflammation altered the fear-related behavior. Previous studies showed that the ventral hippocampus lesion induces a strange behavior changes as a less anxiety-like and less fear behavior and a decrease of the release of corticosterone [27,30]. The baseline of our results showed also no significant differences in anxiety and depression-like behavior in male compared to the female. However, other studies have produced conflicting findings with some studies demonstrating increases [60] in adult-male anxiety-like behavior while others reveal decreases (Walker F.R. et al., 2008). Furthermore, Tenk et al. have found an increase in anxiety-like behavior in male and decrease in the female, which is in contradiction with our findings, a less anxiety-like in male and no significant differences in female (Tenk et al. 2013). Those differences between the results studies may be due to the age of the stimulation, the behavior test used and the experiment conditions [50]. In the current study, the ip injection of $250 \mu \mathrm{g} / \mathrm{kg}$ of LPS in PND14 induced as we report before a less anxiety and depression-like behavior in survived male rats-LPS, while no significant differences are observed in femaleLPS injected. According to the literature, the early activation of Indolamine Dioxygenase (IDO) by pro-inflammatory cytokines alters serotoninergic and glutamatergic neurotransmission [21,34,55] which can induce long-term changes in behavior.

Many studies demonstrated that TNF $\alpha$ signaling plays an important role in the hippocampus and cortex. TNF $\alpha$ appears to regulate hippocampal neuronal development as TNF $\alpha$-deficient mice display accelerated maturation of the DG (Golan Het al.2004). Furthermore, TNF $\alpha$ inhibits glutamate transporters by astrocytes (Marchetti $L$ et al.2004). In addition to the behavior alteration in the adult age, the hippocampal TNF $\alpha$ results showed no significant differences observed between males and females-LPS treated compared to their respective controls in the adult age. While a huge difference was observed between the baselines TNF $\alpha$ displayed by the control female-rat compared to the control male-rat, suggesting that there is no correlation between the current level of TNF $\alpha$ and behavioral changes. The TNF $\alpha$ could alter the behavior during infancy, and plays an important role in neurodevelopment and in the pathophysiology of various neuropsychiatric 
conditions $[14,25,35]$ Schuld et al., demonstrated that the feedback of the HPA and the TNF- $\alpha$ levels, were inversely associated with the ACTH response, and it was concluded that elevated HPA axis activity in acute or chronic depression-like suppresses TNF- $\alpha$ system activity ([22]; Schuld A et al. [41]). The postnatal injection of LPS could then be assimilated to an early life stress which provokes a release of corticosterone and a depletion of the proinflammatory cytokines including the TNF- $\alpha$.

Glial cell-neuronal signaling may also be a key mechanism that influences long-term changes following postnatal LPS exposure.

The inflammation has been shown to lead to immediate and longterm changes in microglia [7]. Until now, it has been shown that under normal developmental conditions, males have more ameboid microglia in the developing brain than females [42]. Since glial cells are involved in the immune response of the brain, the sex-differentiated glial milieu may result in a sex-differentiated response to an inflammatory challenge that may have long-lasting consequences, including sex differences in the number of glial cells in adulthood. In turn, sex differences in the number of glial cells in specific regions of the adult brain may result in sex differences in behavior since glial cells are involved in the regulation of synaptic function [2,33,43,47]. It has been previously described that prenatal administration of LPS results in increased gliosis in different brain regions in adulthood, including the hippocampus $[16,49]$. Our findings indicate that the postnatal PND 14 administration of LPS, has a permanent effect on the number of glial cells in the hippocampus. Thus, male rats that were treated with LPS at PND14 showed an increase in a number of astrocytes in CA1 and CA3, but no changes are observed in DG compared to control animals in dorsal hippocampus. In addition, no significant differences happen in the ventral hippocampus in the male. Contrariwise, the astrocytes numbers increased after postnatal LPS injection in females in all hippocampus areas. Interestingly, in LPS treated animals, the number of astrocytes in these hippocampal regions was higher in females than in males, indicating a sex-dimorphic response to the immune challenge.

The increased number of astroglial cells in female animals treated with LPS was surprising. Since increased gliosis has been associated with anxiety-like and depressive behaviors, especially in the ventral hippocampus. Our results indicate that male injected by LPS has no significant differences compared to their controls [23,56]. The same group has a depression-like behavior, while the females have not shown any significant behavioral alterations. However, the sex differences in the number of glial cells do not necessarily imply sex differences in astrogliosis.

Furthermore, infantile LPS administration caused a significant increase in microglia in dorsal and ventral hippocampal areas in adulthood. Therefore, these findings indicate that an immune challenge during the infantile period has sex-dependent permanent effects on gliosis in adulthood.

Indeed, when the phenotype of microglia was analyzed, males and females showed a similar proportion of activated cells in the dorsal hippocampus. As mentioned before, glial cells participate in the regulation of synaptic function $[2,19,47]$ and release trophic factors for neurons [43]. In this case, the increased number of glial cells observed in females after LPS administration compared to LPS treated males may be inhibited by the estrogen effect and prevent the manifestation of the behavioral alterations observed in males. Several studies reported that, early life immune stimulation is able to activate persistently the microglia and astrocyte from the resting to primed shape, and produce continuous and uncontrolled neurotoxic molecules which can damage the neurones [37]. Taken together, the increase in glial cells number in the dorsal and ventral hippocampus after $>85$ days from the LPS exposition indicate an alteration of this area and that neonatal LPS administration changed the microglia shape in both of sexes and not altered the normal level of TNFa.

The depression-like behavior and the anxiolytic effect may be related to the dorsal hippocampal impairment in the male. In addition, anxiety-like behavior in a female may be due to the ventral hippocampus impairment. Previous studies demonstrated that the dorsal hippocampus is not involved in the affective behavior changes, but maybe in cognitive behavior changes [13]. As mentioned above, activated microglia during infancy promotes expression of inflammatory cytokines that stimulate IDO activity and deplete central nervous system tryptophan which leads to decreased levels of serotonin, and alterations in 5-HT availability result depression-like behavior, especially in the male. The less depression-like behavior induced by LPS in female compared to male, may be due to the estrogen. Many studies have demonstrated that estrogen has the capacity to suppressed IDO level which may suggest that inhibition of IDO release is one of the mechanisms that estrogen preserved the serotonin content [57]. In vitro studies have shown that LPS and estradiol treatment induces different inflammatory signaling responses in male- and female-derived microglia [29]. Sex differences in the inflammatory response of astrocytes to LPS have also been detected in vitro [40].

Additionally, it has been demonstrated that males and females respond differentially to early life infection or bacterial LPS treatment $[5,39]$. Thus, intrauterine LPS administration in rats induces autismlike effects in male but not in female offspring [26] and LPS injection to rat pups causes decreased locomotor activity in adult males, but not in adult females [50].

In summary, our findings indicate that an immune challenge in PND14 in rats induces sex differences in affective behavior in adulthood related to the permanent alteration of the structure and function of the hippocampus immediately after stress exposure by LPS. Furthermore, the hippocampus regions affected by the infancy inflammation are different from male and female, which can determine the type of the behavior that will change at the adult.

\section{Compliance with ethical standards}

All experimental procedures were performed according to the $\mathrm{NIH}$ Guidelines for the Care and Use of Laboratory Animals and under observation and authorization of the doctoral studies center at Ibn Tofail University Kenitra, Morocco.

All the Authors declare that have no conflict of interest.

\section{Acknowledgements}

This research was supported partially by International Brain Research Organization/ARC BURSARIES.

\section{References}

[1] A. Aderem, R.J. Ulevitch, Toll-like receptors in the induction of the innate immune response, Nature 406 (6797) (2000) 782-787, https://doi.org/10.1038/35021228.

[2] A. Araque, M. Navarrete, Glial cells in neuronal network function, Philos. Trans. R. Soc. Lond. Ser. B Biol. Sci. 365 (1551) (2010) 2375-2381, https://doi.org/10.1098/ rstb.2009.0313.

[3] I. Berkiks, H. Benmhammed, A. Mesfioui, A. Ouichou, A. El Hasnaoui, S. Mouden, ... A. El Hessni, Postnatal melatonin treatment protects against affective disorders induced by early-life immune stimulation by reducing the microglia cell activation and oxidative stress, Int. J. Neurosci. (2017) 1-10, https://doi.org/10.1080/ 00207454.2017.1398156.

[4] I.S. Berkiks, L.M. Boulbaroud, A. Garcia-Segura, A. Mesfioui, S. Ouichou, Mouden, ... A.E. Hessni, Thymelaea lythroides extract attenuates microglial activation and depressive-like behavior in LPS-induced inflammation in adult male rats, Biomed. Pharmacother. 99 (2018) 655-663, https://doi.org/10.1016/j.biopha.2018.01. 125.

[5] M.M. Bernardi, L.P. Teixeira, A.P. Ligeiro-De-Oliveira, W. Tavares-De-Lima, J. Palermo-Neto, T.B. Kirsten, Neonatal lipopolysaccharide exposure induces sexually dimorphic sickness behavior in adult rats, Psychol. Neurosci. 7 (2) (2014).

[6] E.W. Bingaman, D.J. Magnuson, T.S. Gray, R.J. Handa, Androgen inhibits the increases in hypothalamic corticotropin-releasing hormone (CRH) and CRH-immunoreactivity following gonadectomy, Neuroendocrinology 59 (3) (1994) 228-234.

[7] S.T. Bland, J.T. Beckley, L.R. Watkins, S.F. Maier, S.D. Bilbo, Neonatal Escherichia coli infection alters glial, cytokine, and neuronal gene expression in response to acute amphetamine in adolescent rats, Neurosci. Lett. 474 (1) (2010) 52-57, 
https://doi.org/10.1016/j.neulet.2010.03.006

[8] L. Boisse, A. Mouihate, S. Ellis, Q.J. Pittman, Long-term alterations in neuroimmune responses after neonatal exposure to lipopolysaccharide, J. Neurosci. 24 (21) (2004) 4928-4934, https://doi.org/10.1523/JNEUROSCI.1077-04.2004.

[9] A. Can, D.T. Dao, M. Arad, C.E. Terrillion, S.C. Piantadosi, T.D. Gould, The mouse forced swim test, J. Vis. Exp. (59) (2012) e3638, https://doi.org/10.3791/3638.

[10] G.P. Chrousos, Stress and sex versus immunity and inflammation, Sci. Signal. 3 (143) (2010) pe36, https://doi.org/10.1126/scisignal.3143pe36.

[11] D. Demaster, C. Johnson, J. Juranek, L. Ewing-Cobbs, Memory and the hippocampal formation following pediatric traumatic brain injury, Brain Behav. 7 (12) (2017) e00832, , https://doi.org/10.1002/brb3.832.

[12] M. Durand, O. Berton, S. Aguere, L. Edno, I. Combourieu, P. Mormede, F. Chaouloff, Effects of Repeated- fluoxetine on Anxiety-Relatedbehaviours, Central Sero- tonergic Systems, and the Corticotropic Axis in SHR and WKY Rats, Neuropharmacology 38 (15) (1999) 893-907.

[13] M.S. Fanselow, H.W. Dong, Are the dorsal and ventral hippocampus functionally distinct structures? Neuron 65 (1) (2010) 7-19, https://doi.org/10.1016/j.neuron. 2009.11.031.

[14] G. Fricchione, R. Daly, M.P. Rogers, G.B. Stefano, Neuroimmunologic influences in neuropsychiatric and psychophysiologic disorders, Acta Pharmacol. Sin. 22 (7) (2001) 577-587.

[15] L.M. Garcia-Segura, M. Duenas, S. Busiguina, F. Naftolin, J.A. Chowen, Gonadal hormone regulation of neuronal-glial interactions in the developing neuroendocrine hypothalamus, J. Steroid Biochem. Mol. Biol. 53 (1-6) (1995) 293-298.

[16] M. Graciarena, V. Roca, P. Mathieu, A.M. Depino, F.J. Pitossi, Differential vulnerability of adult neurogenesis by adult and prenatal inflammation: role of TGF-beta1, Brain Behav. Immun. 34 (2013) 17-28, https://doi.org/10.1016/j.bbi.2013.05. 007.

[17] C.J. Grossman, Regulation of the immune system by sex steroids, Endocr. Rev. 5 (3) (1984) 435-455, https://doi.org/10.1210/edrv-5-3-435.

[18] B. Guardiola-Lemaitre, A. Lenegre, R.D. Porsolt, Combined effects of diazepam and melatonin in two tests for anxiolytic activity in the mouse, Pharmacol. Biochem. Behav. 41 (2) (1992) 405-408.

[19] M.M. Halassa, T. Fellin, P.G. Haydon, Tripartite synapses: roles for astrocytic purines in the control of synaptic physiology and behavior, Neuropharmacology 57 (4) (2009) 343-346, https://doi.org/10.1016/j.neuropharm.2009.06.031.

[20] W.J. Hatton, C.S. von Bartheld, Analysis of cell death in the trochlear nucleus of the chick embryo: calibration of the optical disector counting method reveals systematic bias, J. Comp. Neurol. 409 (2) (1999) 169-186.

[21] E. Herlenius, H. Lagercrantz, Neurotransmitters and neuromodulators during early human development, Early Hum. Dev. 65 (1) (2001) 21-37.

[22] H. Himmerich, E.B. Binder, H.E. Kunzel, A. Schuld, S. Lucae, M. Uhr, ... M. Ising, Successful antidepressant therapy restores the disturbed interplay between TNFalpha system and HPA axis, Biol. Psychiatry 60 (8) (2006) 882-888, https://doi. org/10.1016/j.biopsych.2006.03.075.

[23] M. Hinwood, R.J. Tynan, J.L. Charnley, S.B. Beynon, T.A. Day, F.R. Walker, Chronic stress induced remodeling of the prefrontal cortex: structural re-organization of microglia and the inhibitory effect of minocycline, Cereb. Cortex 23 (8) (2013) 1784-1797, https://doi.org/10.1093/cercor/bhs151.

[24] C. Howard, M. Reed, Unbiased Stereology. Three-Dimensional Measurement in Microscopy, Oxford BIOS Scientific Publishers Limited, 1998.

[25] A. Kelly, E. Vereker, Y. Nolan, M. Brady, C. Barry, C.E. Loscher, ... M.A. Lynch, Activation of p38 plays a pivotal role in the inhibitory effect of lipopolysaccharide and interleukin-1 beta on long term potentiation in rat dentate gyrus, J. Biol. Chem. 278 (21) (2003) 19453-19462, https://doi.org/10.1074/jbc.M301938200.

[26] T.B. Kirsten, M. Taricano, P.C. Maiorka, J. Palermo-Neto, M.M. Bernardi, Prenatal lipopolysaccharide reduces social behavior in male offspring, Neuroimmunomodulation 17 (4) (2010) 240-251, https://doi.org/10.1159/ 000290040.

[27] K.G. Kjelstrup, F.A. Tuvnes, H.A. Steffenach, R. Murison, E.I. Moser, M.B. Moser, Reduced fear expression after lesions of the ventral hippocampus, Proc. Natl. Acad. Sci. U. S. A. 99 (16) (2002) 10825-10830, https://doi.org/10.1073/pnas. 152112399.

[28] K.M. Lenz, M.M. McCarthy, A Starring Role for Microglia in Brain sex differences, Neuroscientist (2014), https://doi.org/10.1177/1073858414536468.

[29] L.C. Loram, P.W. Sholar, F.R. Taylor, J.L. Wiesler, J.A. Babb, K.A. Strand, .. L.R. Watkins, Sex and estradiol influence glial pro-inflammatory responses to lipopolysaccharide in rats, Psychoneuroendocrinology 37 (10) (2012) 1688-1699, https://doi.org/10.1016/j.psyneuen.2012.02.018.

[30] S.B. McHugh, R.M. Deacon, J.N. Rawlins, D.M. Bannerman, Amygdala and ventral hippocampus contribute differentially to mechanisms of fear and anxiety, Behav. Neurosci. 118 (1) (2004) 63-78, https://doi.org/10.1037/0735-7044.118.1.63.

[31] M.M. Moraes, M.C. Galvao, D. Cabral, C.P. Coelho, N. Queiroz-Hazarbassanov, M.F. Martins, ... T.B. Kirsten, Propentofylline Prevents Sickness Behavior and Depressive-like Behavior Induced by Lipopolysaccharide in Rats via Neuroinflammatory Pathway, PLoS One 12 (1) (2017) e0169446, , https://doi.org/ 10.1371/journal.pone.0169446.

[32] S. Munoz-Cruz, C. Togno-Pierce, J. Morales-Montor, Non-reproductive effects of sex steroids: their immunoregulatory role, Curr. Top. Med. Chem. 11 (13) (2011) 1714-1727.

[33] M. Nedergaard, B. Ransom, S.A. Goldman, New roles for astrocytes: redefining the functional architecture of the brain, Trends Neurosci. 26 (10) (2003) 523-530, https://doi.org/10.1016/j.tins.2003.08.008.

[34] J.C. O'Connor, M.A. Lawson, C. Andre, M. Moreau, J. Lestage, N. Castanon, R. Dantzer, Lipopolysaccharide-induced depressive-like behavior is mediated by indoleamine 2,3-dioxygenase activation in mice, Mol. Psychiatry 14 (5) (2009)
511-522, https://doi.org/10.1038/sj.mp.4002148.

[35] K. Okazaki, A. Nishida, M. Kato, K. Kozawa, N. Uga, H. Kimura, Elevation of cytokine concentrations in asphyxiated neonates, Biol. Neonate 89 (3) (2006) 183-189, https://doi.org/10.1159/000089180.

[36] R. Paolicelli, G. Bolasco, F. Pagani, L. Maggi, ... , Synaptic pruning by microglia is necessary for normal brain development, science (2011) 1456-1458.

[37] L. Qin, X. Wu, M.L. Block, Y. Liu, G.R. Breese, J.S. Hong, ... F.T. Crews, Systemic LPS causes chronic neuroinflammation and progressive neurodegeneration, Glia 55 (5) (2007) 453-462, https://doi.org/10.1002/glia.20467.

[38] A. Ramos, O. Berton, P. Mormede, F. Chaouloff, A multiple-test study of anxietyrelated behaviours in six inbred rat strains, Behav. Brain Res. 85 (1) (1997) 57-69.

[39] S.A. Rana, T. Aavani, Q.J. Pittman, Sex effects on neurodevelopmental outcomes of innate immune activation during prenatal and neonatal life, Horm. Behav. 62 (3) (2012) 228-236, https://doi.org/10.1016/j.yhbeh.2012.03.015.

[40] M. Santos-Galindo, E. Acaz-Fonseca, M.J. Bellini, L.M. Garcia-Segura, Sex differences in the inflammatory response of primary astrocytes to lipopolysaccharide, Biol. Sex Differ. 2 (2011) 7, https://doi.org/10.1186/2042-6410-2-7.

[41] A. Schuld, D.A. Schmid, M. Haack, F. Holsboer, E. Friess, P. T, Hypothalamo pituitary adrenal function in patients with depressive disorders is correlated with baseline cytokine levels but not with cytokine responses to hydrocortisone, J. Psychiatr. Res. 37 (2003) 463-470.

[42] J.M. Schwarz, P.W. Sholar, S.D. Bilbo, Sex differences in microglial colonization of the developing rat brain, J. Neurochem. 120 (6) (2012) 948-963, https://doi.org/ 10.1111/j.1471-4159.2011.07630.x.

[43] A. Sharif, V. Prevot, ErbB receptor signaling in astrocytes: a mediator of neuron-glia communication in the mature central nervous system, Neurochem. Int. 57 (4) (2010) 344-358, https://doi.org/10.1016/j.neuint.2010.05.012.

[44] L. Shi, S.H. Fatemi, R.W. Sidwell, P.H. Patterson, Maternal influenza infection causes marked behavioral and pharmacological changes in the offspring, J. Neurosci. 23 (1) (2003) 297-302.

[45] S.J. Spencer, M.A. Galic, Q.J. Pittman, Neonatal programming of innate immune function, Am. J. Physiol. Endocrinol. Metab. 300 (1) (2011) E11-E18, https://doi org/10.1152/ajpendo.00516.2010.

[46] E. Spinedi, N. Perez, R. Gaillard, Neuroendocrine-Immune Interactions: Impact of Gender, Negative Energetic Balance Condition, and Developmental Stage on the Mechanisms of the Acute Phase Response of the Inflammatory Process in Mammals, (2004), pp. 303-354.

[47] J. Stipursky, L. Romao, V. Tortelli, V.M. Neto, F.C. Gomes, Neuron-glia signaling: Implications for astrocyte differentiation and synapse formation, Life Sci. 89 (15-16) (2011) 524-531, https://doi.org/10.1016/j.lfs.2011.04.005.

[48] B.J. Stoll, N.I. Hansen, I. Adams-Chapman, A.A. Fanaroff, S.R. Hintz, B. Vohr, R.D. Higgins, Neurodevelopmental and growth impairment among extremely low birth weight infants with neonatal infection, JAMA 292 (19) (2004) 2357-2365.

[49] E. Strackx, M. Gantert, V. Moers, I.A. van Kooten, R. Rieke, H. Hurter, B.W. Kramer, Increased number of cerebellar granule cells and astrocytes in the internal granule layer in sheep following prenatal intra-amniotic injection of lipopolysaccharide, Cerebellum 11 (1) (2012) 132-144, https://doi.org/10.1007/ s12311-011-0297-7.

[50] C.M. Tenk, M. Kavaliers, K.P. Ossenkopp, Sexually dimorphic effects of neonatal immune system activation with lipopolysaccharide on the behavioural response to a homotypic adult immune challenge, Int. J. Dev. Neurosci. 26 (3-4) (2008) 331-338, https://doi.org/10.1016/j.ijdevneu.2008.01.001.

[51] N.C. Vamvakopoulos, G.P. Chrousos, Evidence of direct estrogenic regulation of human corticotropin-releasing hormone gene expression. Potential implications fo the sexual dimophism of the stress response and immune/inflammatory reaction, J. Clin. Invest. 92 (4) (1993) 1896-1902, https://doi.org/10.1172/JCI116782.

[52] M.M. van Gaalen, T. Steckler, Behavioural analysis of four mouse strains in an anxiety test battery, Behav. Brain Res. 115 (1) (2000) 95-106.

[53] K.C. Wang, L.W. Fan, A. Kaizaki, Y. Pang, Z. Cai, L.T. Tien, Neonatal lipopolysaccharide exposure induces long-lasting learning impairment, less anxiety-like response and hippocampal injury in adult rats, Neuroscience 234 (2013) 146-157, https://doi.org/10.1016/j.neuroscience.2012.12.049.

[54] Weibel, Practical Methods for Biological Morphometry, Academic Press, 1979.

[55] L. Wischhof, E. Irrsack, C. Osorio, M. Koch, Prenatal LPS-exposure-a neurodevelopmental rat model of schizophrenia-differentially affects cognitive functions, myelination and parvalbumin expression in male and female offspring, Prog. NeuroPsychopharmacol. Biol. Psychiatry 57 (2015) 17-30, https://doi.org/10.1016/j. pnpbp.2014.10.004.

[56] E.S. Wohleb, M.L. Hanke, A.W. Corona, N.D. Powell, L.M. Stiner, M.T. Bailey, ... J.F. Sheridan, Beta-Adrenergic receptor antagonism prevents anxiety-like behavior and microglial reactivity induced by repeated social defeat, J. Neurosci. 31 (17) (2011) 6277-6288, https://doi.org/10.1523/JNEUROSCI.0450-11.2011.

[57] Y. Xu, H. Sheng, Z. Tang, J. Lu, X. Ni, Inflammation and increased IDO in hippocampus contribute to depression-like behavior induced by estrogen deficiency, Behav. Brain Res. 288 (2015) 71-78, https://doi.org/10.1016/j.bbr.2015.04.017.

[58] H.M. Yu, T.M. Yuan, W.Z. Gu, J.P. Li, Expression of glial fibrillary acidic protein in developing rat brain after intrauterine infection, Neuropathology 24 (2) (2004) $136-143$

[59] Y. Zhan, R.C. Paolicelli, F. Sforazzini, L. Weinhard, G. Bolasco, F. Pagani, C.T. Gross, Deficient neuron-microglia signaling results in impaired functional brain connectivity and social behavior, Nat. Neurosci. 17 (3) (2014) 400-406, https:// doi.org/10.1038/nn.3641.

[60] C. Walker, A. Sunderland, J. Sharma, M. Walker, The impact of cognitive impairment on upper body dressing difficulties after stroke: a video analysis of patterns of recovery, J. Neurol. Neurosurg. Psychiatry. 75 (1) (2004 Jan) 43-48. 